\title{
BMJ Open Effective SLOPE: EffectS of Lifestyle interventions in Older PEople with obesity: a systematic review and network meta-analysis protocol
}

\author{
Gabriel Torbahn (D) , ${ }^{1}$ Daniel Schoene (D) , ${ }^{2}$ Lukas Schwingshackl (D) , ${ }^{3}$ \\ Gerta Rücker (1) , ${ }^{4}$ Helge Knüttel (D) , ${ }^{5}$ Wolfgang Kemmler (D) , \\ Cornel C Sieber (1D , 1,7 John A Batsis (D) , ${ }^{8,9}$ Dennis T Villareal (D) ,10 \\ Nanette Stroebele-Benschop (D) , ${ }^{11}$ Dorothee Volkert (D) , ${ }^{1}$ Eva Kiesswetter (D) ${ }^{1}$
}

To cite: Torbahn G, Schoene D, SchwingshackI L, et al. Effective SLOPE: EffectS of Lifestyle interventions in Older PEople with obesity: a systematic review and network metaanalysis protocol. BMJ Open 2020;10:e038330. doi:10.1136/ bmjopen-2020-038330

- Prepublication history and supplemental material for this paper are available online. To view these files, please visit the journal online (http://dx.doi. org/10.1136/bmjopen-2020038330).

Received 06 March 2020 Revised 12 August 2020 Accepted 03 September 2020

Check for updates

(C) Author(s) (or their employer(s)) 2020. Re-use permitted under CC BY-NC. No commercial re-use. See rights and permissions. Published by BMJ.

For numbered affiliations see end of article.

Correspondence to Mr Gabriel Torbahn; gabriel.torbahn@fau.de

\section{ABSTRACT}

Introduction Obesity is highly prevalent in older adults aged 65 years or older. Different lifestyle interventions (diet, exercise, self-management) are available but benefits and harms have not been fully quantified comparing all available health promotion interventions. Special consideration must be given to functional outcomes and possible adverse effects (loss of muscle and bone mass, hypoglycaemia) of weight loss interventions in this age group. The objective of this study is to synthesise the evidence regarding the effects of different types and modalities of lifestyle interventions, or their combinations, on physical function and obesity-related outcomes such as body composition in older adults with obesity.

Methods and analyses Six databases (Medline, Embase, Cochrane Central Register of Controlled Trials, Cumulated Index to Nursing and Allied Health Literature (CINAHL), Psychinfo and Web of Science) and two trial registries (Clinicaltrials.gov and the WHO International Clinical Trials Registry Platform) will be searched for randomised controlled trials of lifestyle interventions in older adults with obesity. Screening (title/abstract and full-text) and data extraction of references as well as assessment of risk of bias and rating of the certainty of evidence (Grading of Recommendations, Assessment, Development and Evaluation for network meta-analyses) will be performed by two reviewers independently. Frequentist randomeffects network meta-analyses will be conducted to determine the pooled effects from each intervention. Ethics and dissemination We will submit our findings to peer-reviewed journals and present at national and international conferences as well as in scientific medical societies. Patient-targeted dissemination will involve local and national advocate groups.

PROSPERO registration number CRD42019147286.

\section{INTRODUCTION}

Obesity is defined as an abnormal and excessive accumulation of body fat, ${ }^{1}$ while on a population level, is defined using a body mass index $(\mathrm{BMI}) \geq 30 \mathrm{~kg} / \mathrm{m}^{2}$. Over the past four decades, the prevalence of obesity has been

\section{Strengths and limitations of this study}

- This will be the first network meta-analyses (NMA) on lifestyle interventions in older adults with obesity.

Rather than focussing on weight loss, physical functioning will be the primary interest due to its subjective and objective relevance for older people.

- Methods will be applied based on the standards of the updated version 6.0 of the Cochrane Handbook for Interventions (updated July 2019).

- Recommendations will be derived based on the results according to the Grading of Recommendations, Assessment, Development and Evaluation approach for NMA.

- Heterogeneity (clinical and statistical) will be evaluated and discussed in detail.

increasing worldwide across all age groups. ${ }^{2}$ The rate of obesity in older adults, the fastest growing population segment, ${ }^{3}$ have now exceeded $40 \%$, making this a public health concern. ${ }^{4}$ As BMI has poor sensitivity in older adults due to age-related changes in body composition and a reduction of body height, ${ }^{5}$ waist circumference and, more directly, objectively-measured fat mass can be considered in ascertaining obesity. In the USA, central obesity measured using waist circumference has been found in $\sim 63 \%$ of community-dwelling adults aged $\geq 60$ years. ${ }^{6}$ The prevalence of obesity according to a high proportion of fat mass is $64 \%$ and $77 \%$ in German women and men $\geq 70$ years, respectively. ${ }^{78}$ Due to the higher mechanical load of a higher body weight, for a long time obesity has not been linked to a low proportion of muscle mass. However, in recent years, sarcopenic obesity, a syndrome combining obesity with low muscle mass and strength or physical function, has gained considerable attention. 
Sarcopenic obesity is a largely underdiagnosed condition in clinical practice, and prevalences of up to $94 \%$ in older adults depending on the operationalisation of this construct have been reported. ${ }^{9}$

In community-dwelling older adults, obesity and sarcopenic obesity are associated with increased mortality ${ }^{10} 11$ as well as with reduced quality of life (QoL) ${ }^{1213}$ Contrary, several cohort studies have shown a lower risk for mortality in people with obesity and specific diseases such as type 2 diabetes, coronary artery disease or serious illnesses, ${ }^{14-16}$ which was described as 'obesity paradox'. While research on this controversial phenomenon is still ongoing, several hypotheses are discussed, such as collider bias or effect modification. ${ }^{17-19}$ Obesity is a well-known risk factor for metabolic and cardiovascular diseases, pulmonary abnormalities and certain types of cancer in older age.$^{20}$ Furthermore, obesity is associated with the onset of osteoarthritis in older adults, ${ }^{21}$ one of the most disabling medical conditions, severely affecting one's QoL. ${ }^{22}$ A meta-analysis of 26 prospective studies in older adults revealed obesity as risk factor for functional decline ${ }^{23}$ which is of utmost importance for independent living. ${ }^{24}{ }^{25}$ Older adults with sarcopenic obesity are considered a group at particular risk for functional limitations as they are suffering from two conditions determining functional disability simultaneously. ${ }^{26}{ }^{27}$ Moreover, in older people obesity and sarcopenic obesity are associated with an increased risk of falls ${ }^{28-31}$ and nursing home admissions. ${ }^{32}$ Alley et al have predicted that given the increasing prevalence of obesity, a disabled older person with obesity may become the most common phenotype of frailty ${ }^{33}$-another syndrome in the geriatric population that is associated with decline in health and function ${ }^{34}{ }^{35}$ - posing a marked personal and societal burden. In 2015, a high BMI contributed to about 120 million disability-adjusted life years (DALYs) representing $\sim 5 \%$ of DALYs from any causes among adults worldwide. ${ }^{2}$ A recent systematic review found that compared with healthy weight, the total annual healthcare costs are 30\% (IQR: 20\%-34\%) higher in middleaged and older people with obesity. ${ }^{36}$ An analysis of the World Obesity Foundation in 2017 has forecasted that costs of consequences of overweight and obesity will further increase in the future. ${ }^{37}$

Although other therapeutic options to treat obesity exist (eg, bariatric surgery), lifestyle strategies should always be first-line treatment. ${ }^{38-40}$ Lifestyle interventions mainly focus on diet, exercise, self-management or combined strategies that vary in treatment modality (eg, specific content), type of delivery (eg, level of supervision) and dose. Lifestyle interventions mainly focus on diet (eg, calorie restriction ${ }^{41}$ ), high-protein diet, ${ }^{42}$ exercise (eg, aerobic or resistance ${ }^{43}$ ), self-management interventions (eg, relapse prevention or self-monitoring techniques ${ }^{44}$ ) or combined strategies that vary in treatment modality (eg, specific content), type of delivery (eg, level of supervision, individual vs group sessions, in person vs technology) and dose (eg, duration, intensity). Findings from younger people cannot be generalised to older people due to higher levels of multimorbidity, frailty, sarcopenia and malnutrition risk. ${ }^{45}$ Moreover, harmful side effects of interventions aiming at weight loss have to be considered, such as reduced muscle mass ${ }^{46}$ and bone mineral density. ${ }^{47}$ Thus, in older people functional decline, functional limitations as well as the risk of adverse events, such as falls and fractures, may be increased. ${ }^{48}$ Very low caloric diets may lead to an inadequate intake of nutrients and consequently to the development of malnutrition, another geriatric syndrome associated with adverse health events. ${ }^{49}$ In addition, perceived and actual barriers differ between younger and older adults in their impact on adopting lifestyle changes. ${ }^{50}$ Despite these issues, obesity treatment in older adults is still not sufficiently addressed in existing obesity guidelines. ${ }^{39} 5152$

Several systematic reviews on obesity treatment in older adults have been published between 2006 and $2019^{53-63}$ including 126 publications of more than 60 distinct randomised controlled trials (RCTs). These systematic reviews, however, did not identify the same studies for inclusion due to different search strategies, databases, search dates as well as differing definitions of obesity and applying various age cut-offs. They generally agree that weight-loss interventions in older adults do not cause poor health outcomes (eg, higher risk for mortality for those randomised to the weight-loss group and significantly reduce weight). Further, more limited evidence demonstrates improvements in measures of physical performance, such as gait speed. Combined interventions (eg, including dietary and exercise components) are to be favoured to preserve muscle mass, bone mineral density and to improve physical performance. However, self-management strategies, which are important for long-term weight maintenance from studies in younger adults ${ }^{64}$ have not been separately reported and discussed in existing reviews on the management of obesity in older adults. In addition, methodological issues prevent the drawing of firm conclusions, for example, recommendations for obesity treatment. These include too specific searches in only one database, not covering the complete time period of databases and application of language restrictions. This must be considered insufficient as it likely missed relevant evidence. ${ }^{65}{ }^{66}$ Further, a quality rating of the included RCTs was missing in the majority of these systematic reviews or when done, some used no standardised tools. The only published meta-analysis dates back to $2010,^{63}$ and there is no meta-analysis available for functional outcomes in older people with obesity. Considering, recently published intervention studies, for example, Ard et $a l^{67}$ and Beavers et $a l^{68}$ it is likely that accumulated evidence enables quantitative syntheses.

These limitations of existing systematic reviews highlight an evidence gap and justify the need for a thoroughly conducted high-quality systematic review according to the updated standards described by the Cochrane collaboration for network meta-analysis. ${ }^{69} 70$ As older adults are particularly susceptible to negative effects of excess body mass on physical function due to the age-related decline 
in muscle mass and strength ${ }^{20}$ and frequently report the priority of functional outcomes related to mobility and daily life tasks, ${ }^{71}$ these outcomes should be investigated comprehensively.

An important question remaining is which type of lifestyle intervention or treatment modality offers optimal benefits in older adults with obesity. As there exist a large number of possible interventions, multiple pairwise metaanalyses are insufficient to provide an answer of high certainty. Therefore, we will conduct a comprehensive systematic review with network meta-analyses (NMA) of RCTs to synthesise the evidence regarding the beneficial and potentially harmful effects of different types and modalities of lifestyle interventions, or their combinations, on physical function and obesity-related outcomes such as body composition in older adults with obesity and sarcopenic obesity.

\section{METHODS AND ANALYSIS \\ Reporting}

We report this protocol according to the Preferred Reporting Items for Systematic Review and Meta-Analyses statement for systematic review protocols (PRISMA-P, see online supplemental file 1), ${ }^{72}$ the additional guidance for NMA by Chaimani $e t a l^{33}$ and the guidance for systematic reviews of older adults by Shenkin $e t a l^{74}$ to ensure thorough reporting and implementation. The methodology is preregistered on the International Prospective Register of Systematic Reviews (registration number CRD42019147286). ${ }^{75}$

\section{Eligibility criteria}

We will select primary studies according to the criteria below.

\section{Population}

To focus this systematic review on older adults, we will include studies including adults with a minimum age of 60 years and a mean of $\geq 65$ years. ${ }^{76}$ Participants will be classified as obese if one of the following criteria is fulfilled: percentage of total body fat mass $\geq 35 \%$ and $\geq 25 \%{ }^{77}$ or waist circumference of $\geq 88 \mathrm{~cm}$ and $\geq 102 \mathrm{~cm}$ for women and men, ${ }^{78}$ or BMI, applying the standard adult cut-off of $\geq 30 \mathrm{~kg} / \mathrm{m}^{2}$ since there is no consensus on age-adjusted cut-offs. ${ }^{79}$ If proven valid, we will, however, consider different cut-off values for these criteria, for example, in Asian populations. For all three operationalisations, the methods of measurement applied by individual studies will be used. When studies report mixed samples of older adults with overweight and obesity, we will contact the authors to request the data for the subgroup with obesity. If the provision of data is not possible, the study will be excluded. No consensus definition of sarcopenic obesity exists and various operationalisations are in use. ${ }^{9}$ As such, the definition applied by the primary study will be used, and we shall acknowledge differences in potential subgroup or sensitivity analyses, if possible. Due to the high prevalence of multimorbidity in older people and existing obesity-related comorbidities, participants with common comorbidities of obesity (eg, diabetes, cardiovascular disease, metabolic syndrome, chronic kidney disease, osteoarthritis and geriatric syndromes (eg, frailty and sarcopenia)) will be included. We will only include studies comprising community-dwelling older adults, due to the predictive value of obesity for nursing home admissions. ${ }^{80}$ Studies focusing on animals, genetics or biochemistry will be excluded. References that have not been included after full-text screening will be listed in a table with the respective reason(s) for exclusion.

\section{Interventions}

We will include any type of lifestyle intervention, for example, diet, exercise, self-management, as well as all treatment modalities and their combinations with all types of deliveries and doses. For the dietary component, interventions affecting energy balance, such as energy restriction, balanced (healthy) diet (eg, food pyramid), Mediterranean diet, high-protein diet, lowfat diet, moderate-carbohydrate diet, low-carbohydrate diet, low glycaemic index/glycaemic load diet, vegetarian diet, Dietary Approaches to Stop Hypertension (DASH), will be considered. Interventions providing only micronutrient supplements (eg, vitamin D) as well as studies using only very low energy diets $(<800 \mathrm{kcal} /$ day $)$ or total diet replacement will be excluded. ${ }^{81}$ Additionally, RCTs focussing on substances such as secondary plant products (eg, polyphenols), components of macronutrients (eg, fatty (docosahexaenoic acid) or amino acids (eg, leucin)) and fibres will also be excluded. The exercise component will be defined as any planned, structured and repetitive movement with the objective to improve or maintain physical fitness, for example, aerobic, resistance, balance training, according to the definition of the American College of Sports Medicine. ${ }^{82}$ We will also consider physically supported methods, such as electrical muscle stimulation and vibration training when combined with gross movements or done in an upright position. Finally, as recommended in obesity guidelines ${ }^{83}$ we will include all self-management interventions that intend to support behaviour changes (such as motivational interviewing, social support, cognitive-therapeutic intervention). ${ }^{84}$ This is owed to the fact that many (older) people with chronic diseases (such as diabetes or obesity) have difficulties to control intended behavioural changes (such as improving eating behaviour, increasing physical activity and decreasing sedentary time) ${ }^{85}$ In addition, self-efficacy, self-regulation skills were found important mediators for successful weight change. ${ }^{86}$

\section{Comparators}

Since NMA will be conducted, all interventions will be compared with each other. Additionally, control groups, such as usual care or health counselling, will be considered as comparators. 


\section{Outcomes}

Only previously validated outcomes will be considered and need to be measured at least preintervention and postintervention.

\section{Main outcome}

The change in functional status with focus on physical function was shown to be important to health and adverse outcomes $^{2425}$ and patient-relevant ${ }^{71}$ and will therefore be our main outcome. This includes standard measures of strength, mobility and functional performance for independence in daily living, including their modifications. Common measurements include but are not limited to one-leg stance (balance), gait speed (gait, mobility), 6 min walk test (endurance), repeated chair stands (functional strength, lower extremity function), grip strength (strength, overall function), leg power as well as composite scores of functional tests such as the shortphysical-performance battery ${ }^{25}$ or the physical performance test. ${ }^{87}$ Patient-reported outcomes of functional status (eg, Late-Life Function and Disability Instrument) and digital measurements (eg, instrumented gait analysis)) will also be considered.

\section{Other outcomes}

To evaluate changes in weight and body composition, we will consider measures such as total body mass, fat mass (eg, total, central, peripheral), lean mass, muscle mass (eg, total, appendicular, lower extremity skeletal), bone mineral density (eg, hip, lumbar spine, whole body).

(Health-related) QoL will be summarised when reported by standardised instruments such as 36-item short form survey $^{88}$ or EuroQol-5D. ${ }^{89}$ If reported in primary studies, emotional status (eg, depressive symptoms, depression), social participation (eg, informal social relationships, community life) and satisfaction with intervention will also be captured.

Data on the occurrence of mortality, falls, fractures, hospital admission and nursing home placement as well as for other health-related event data (eg, hypoglycaemia, hypotension), no matter if reported as outcome or adverse event, will also be considered for the current analysis.

\section{Design of primary studies}

We will include (quasi-) RCTs (parallel and crossover). Due to a lower level of initial fitness, prevalent health restrictions and the time needed to respond to treatment, we will include studies with intervention durations of $\geq 12$ weeks. $^{50}$

We will not set any restrictions regarding language or time frame. We will involve colleagues who are fluent in the respective languages or use online translators (eg, https://www.deepl.com/home).

Conference abstracts will be excluded.

\section{Search strategy}

Six electronic databases (Medline, Embase, Cochrane Central, Cumulated Index to Nursing and Allied Health
Literature (CINAHL), PsychInfo and Web of Science) for published trials and two trial registries (Clinicaltrials. gov, WHO International Clinical Trials Registry Platform) for unpublished or ongoing trials will be searched. We developed the search strategy for Medline (via Ovid) (see online supplemental file 2) using a search block for people aged $\geq 65$ years and adapted a block for interventions from a recently published Cochrane review evaluating lifestyle interventions in paediatric patients with overweight and obesity, which was reviewed and revised by information specialists. ${ }^{90}$ For other databases, the search strategy will be adapted according to the database-specific requirements. Additionally, we will screen reference lists of published systematic reviews and eligible RCTs for potential consideration of further primary RCTs and will contact the advisory board which consists of clinical and scientific experts to enquire whether all relevant studies were identified.

\section{Selection process}

Identified references will be saved in Endnote and after excluding duplicates, references will be uploaded to Covidence (http://www.covidence.org). Two reviewers (GT, DS) will independently screen titles/abstracts and full texts for eligibility according to the criteria described above. The title/abstract screening will be piloted using the first 200 references and in case of too many deviations $(>10 \%)$, it will be revised. Disagreements will be solved by discussion or if no consensus can be reached by a third reviewer who will be asked based on his/her expertise (nutrition/general (EK), exercise (WK), selfmanagement (NS-B)). If relevant information is lacking, we will contact the corresponding author/s twice at a weekly interval.

\section{Data extraction}

Two reviewers (GT, DS) will extract data of included references independently using a piloted data extraction table. In case of no consensus, a third reviewer (based on expertise) will solve disagreements. If relevant data are missing, we will contact the corresponding author/s twice at weekly intervals.

When extracting the data, we will consider the following information: study characteristics: for example, author, publication year, eligibility criteria, setting, study duration, sample size, follow-up time, conflict of interest; participants' characteristics: for example, age, sex, ethnicity, BMI, body composition (eg, fat mass, muscle mass, height/ weight adjusted indices), comorbidities (eg, diabetes, cardiovascular disease), geriatric syndromes (eg, sarcopenia, frailty, cognitive impairment), functional status, lifestyle behaviour (eg, sedentary); intervention characteristics: type and modality, type of delivery, dose (eg, duration, frequency, intensity), control arms, cointerventions, compliance and adherence, drop out, (serious) adverse events related to intervention; outcomes: baseline values and follow-up values of functional status, BMI, weight, body composition (lean mass, fat mass), QoL, emotional 
status, social participation and any reported poor health outcome as reported by study authors.

\section{Assessment of risk of bias}

The risk of bias will be assessed after a pilot trial $(n=3)$ by two reviewers (GT, DS; not blinded to authors and journal of primary studies) independently using the revised Cochrane risk of bias tool (RoB 2.0) for RCTs. ${ }^{91} 92$ According to this, sources of bias will be identified by assessing: (1) the randomisation process, (2) deviations from intended interventions, (3) missing outcome data, (4) measurement of the outcome and (5) selection of the reported result. For each domain, available algorithms will be followed to answer the signalling questions (response options: yes, probably yes, probably no, no or no information) and to judge the risk of bias as low, some concerns, or high. The overall risk of bias will also be rated as low (if low risk of bias in all domains), some concerns (at least one domain is rated as having some concerns but no domain is rated by a high risk of bias) or high (if at least one domain is judged with a high risk of bias or multiple domains are rated as having some concerns which might impact the confidence in a result). We will present results in a risk of bias summary graph.

\section{Assessment of certainty of evidence}

Grading of Recommendations, Assessment, Development and Evaluation approach for NMA will be used to assess the certainty of evidence. ${ }^{93-95}$ In addition to the risk of bias rating for every outcome, this includes the rating of direct and indirect evidence for inconsistency, indirectness and dissemination bias. In case of high certainty and a similar contribution of direct and indirect evidence to the network estimate, the highest rating will be used but could be further downrated for incoherence and imprecision. In case of insufficient evidence as well as moderate, low or very low certainty, the indirect estimate will be rated by the lowest of two direct comparisons included in first-order loops and could be further downrated for intransitivity. Dissemination bias will be investigated by searching for unpublished trials (see section search strategy). 'Summary of findings' tables adapted for NMA results will be presented, similar to the proposal by YepesNuñez et $a l^{96}$

\section{Statistical analyses}

Measures of treatment effect

Effect sizes for continuous outcomes (eg, weight loss, muscle strength) will be expressed as mean difference or standardised mean difference with $95 \%$ CI. For dichotomous outcomes (eg, negative health outcome such as death), effect sizes will be expressed as risk ratios with $95 \%$ CI. $^{97}$ In exceptional cases (ie, if a minor number of RCTs expressed as negative health outcome continuously while the majority used dichotomous outcomes), the outcomes reported as continuous or categorical will be dichotomised. ${ }^{98}$ If the postintervention values with the corresponding SD are not available, the changed scores with the corresponding SD will be used. ${ }^{99}$

\section{Data synthesis}

We will conduct random effects model NMA based on a frequentist approach to derive pooled estimates for all outcomes. ${ }^{100}$ We will use the $\mathrm{R}$ package 'netmeta'. ${ }^{101}$ In NMA, evidence from direct comparisons and indirect comparisons is averaged to calculate a network estimate. The key requirement for conducting NMA is that the transitivity assumption-to compare two interventions via an indirect route in the network-is ensured. We assume that for our planned analyses, all interventions are jointly randomisable ${ }^{73102}$ and that all participants are likely to receive any kind of included interventions. Network graphs will be generated by function netgraph() of netmeta. ${ }^{01} 103$ We will assess global incoherence by decomposing the $Q$ statistic into heterogeneity (within designs) and inconsistency (between designs) and visualise this using a net-heat plot. ${ }^{104}$ In addition, we will report and assess inconsistency by calculating differences between direct and indirect effect estimates using descriptive z-tests (function netsplit ()) and report the distribution of direct and indirect evidence. The treatment modalities (eg, very low caloric diet, aerobic exercise, their combination or no intervention (eg, health counselling, healthy eating/ exercise advise)) will build the nodes of the network providing maximising similarity within and minimising similarity between the nodes ${ }^{93}$ To further identify important determinants of efficacy and safety, nodes will be further defined, for example, according to the duration, intensity, mode of delivery of interventions. ${ }^{105}$ Based on data availability, these nodes will be defined after data extraction. Additionally, we will analyse the components (eg, of combined interventions using an additive model for multicomponent interventions). ${ }^{106}$ Models of this type allow disentangling the effects of all single components (eg, very low caloric diet (A), aerobic exercise (B), behavioural group counselling (C)) of a multicomponent intervention arm consisting of at least two single components (eg, $\mathrm{A}+\mathrm{B}, \mathrm{A}+\mathrm{C}, \mathrm{B}+\mathrm{C}$ or $\mathrm{A}+\mathrm{B}+\mathrm{C})$. Since we do not believe that lifestyle interventions that are available for treatment of obesity may fulfil the additivity assumption for component NMA (CNMA) - that is, the effect of a multicomponent intervention equals the sum of their components without any interactions-we will use the interaction CNMA model which is implemented in the function netcomb () of netmeta. ${ }^{101} 106$ In the case of disconnected networks, we will reconnect the networks if possible (ie, presence of at least one common component in the subnetworks). This feature of CNMA is also implemented in netmeta (function $\operatorname{discomb}()$ ).

A secondary data analysis will be conducted using intervention types as network nodes (eg, diet, exercise, selfmanagement). Results of NMA will be presented as forest plots. We will present league tables containing relative treatment effects for all direct comparisons (function netleague ()$)$ and a ranking of all treatments by P-scores. ${ }^{107}$ 


\section{Sensitivity and subgroup analyses}

If possible, sensitivity analyses will be conducted by only including studies rated as low risk of bias. We will try to conduct subgroup analyses for type of obesity (sarcopenic obesity vs obesity), intervention duration $(</>6$ months), age $(</>75$ years $)$, sex, BMI group $\left(</>35 \mathrm{~kg} / \mathrm{m}^{2}\right)$ and comorbidities, such as diabetes or metabolic syndrome and frailty status. Patients' characteristics for subgroup analysis were selected based on the assumption that lifestyle interventions might work differently in people who differ in aspects like vulnerability, resilience and body composition.

\section{Patient and public involvement}

Before the start of this NMA, we have conducted and are currently analysing a qualitative study with semistructured interviews in older persons with obesity. The aim is to obtain further information on patients' motives, barriers, experiences and perceptions regarding therapeutic lifestyle interventions and thus, potentially identify evidence gaps. The results will be published in a separate manuscript.

In addition, we discuss patient-relevant outcomes, existing obstacles that exacerbate the process of contacting this population of patients as well as potential dissemination strategies with representatives of German patient advocate groups.

\section{ETHICS AND DISSEMINATION}

For NMA, there is no direct data collection from human participants and hence, no ethical approval is necessary.

We will submit our research articles to peer-reviewed journals and will present our results at national and international conferences. Involved experts will disseminate the results in scientific and medical societies. We will further disseminate our project via partner universities' websites and press releases. Patient-targeted dissemination will involve local and national advocate groups and offices for senior affairs. In addition, we will disseminate the results by distribution of materials in plain language.

\section{Author affiliations}

${ }^{1}$ Institute for Biomedicine of Aging, Friedrich-Alexander-Universität Erlangen-

Nürnberg, Nürnberg, Bayern, Germany

${ }^{2}$ Institute of Medical Physics, Friedrich-Alexander-Universität Erlangen-Nürnberg, Erlangen, Bayern, Germany

${ }^{3}$ Institute for Evidence in Medicine, Faculty of Medicine, Medical Center - University of Freiburg, Freiburg, Germany

${ }^{4}$ Institute of Medical Biometry and Medical Informatics, Faculty of Medicine, Medical Center - University of Freiburg, Freiburg, Germany

${ }^{5}$ University Library, University of Regensburg, Regensburg, Germany

${ }^{6}$ Institute of Medical Physics, University of Erlangen-Nürnberg, Erlangen, Germany ${ }^{7}$ Department of Medicine, Kantonsspital Winterthur, Winterthur, Zurich, Switzerland ${ }^{8}$ Division of Geriatric Medicine, School of Medicine, University of North Carolina at Chapel Hill, Chapel Hill, North Carolina, USA

${ }^{9}$ Department of Nutrition, Gillings School of Global Public Health, University of North Carolina at Chapel Hill, Chapel Hill, North Carolina, USA

${ }^{10}$ Division of Endocrinology, Diabetes and Metabolism, Baylor College of Medicine, Houston, Texas, USA

${ }^{11}$ Department of Nutritional Psychology, Institute of Nutritional Medicine, University of Hohenheim, Stuttgart, Germany
Acknowledgements The authors thank the information specialist Edith Motschall for reviewing the search strategy. The present work was performed in partial fulfillment of the requirements for obtaining the degree Dr. rer. Biol. hum (Doctoral Degree in Human Biology) for GT.

Contributors GT, DS and EK planned and designed this project, drafted the manuscript and approved the final version. LS, GR, HK, WK, CCS, JAB, DTV, NS-B, and DV were involved in the planning and design process of this project, provided critical feedback for the manuscript and approved the final version. EK will be the guarantor of the review.

Funding This work was funded by the German Federal Ministry of Education and Research (BMBF) grant number (01KG1903).

Competing interests $L S$ is a member of the GRADE working group. JAB is funded by the National Institute on Aging and Office of Dietary Supplements of the National Institutes of Health under Award Number K23AG051681, and the R01 AG067416.

Patient and public involvement Patients and/or the public were involved in the design, or conduct, or reporting, or dissemination plans of this research. Refer to the Methods section for further details.

Patient consent for publication Not required.

Provenance and peer review Not commissioned; externally peer reviewed.

Open access This is an open access article distributed in accordance with the Creative Commons Attribution Non Commercial (CC BY-NC 4.0) license, which permits others to distribute, remix, adapt, build upon this work non-commercially, and license their derivative works on different terms, provided the original work is properly cited, appropriate credit is given, any changes made indicated, and the use is non-commercial. See: http://creativecommons.org/licenses/by-nc/4.0/.

\section{ORCID iDs}

Gabriel Torbahn http://orcid.org/0000-0003-1463-3119

Daniel Schoene http://orcid.org/0000-0003-0717-5746

Lukas Schwingshackl http://orcid.org/0000-0003-3407-7594

Gerta Rücker http://orcid.org/0000-0002-2192-2560

Helge Knüttel http://orcid.org/0000-0002-2654-6517

Wolfgang Kemmler http://orcid.org/0000-0003-3515-0669

Cornel C Sieber http://orcid.org/0000-0002-6271-7459

John A Batsis http://orcid.org/0000-0002-0845-4416

Dennis T Villareal http://orcid.org/0000-0003-1365-7960

Nanette Stroebele-Benschop http://orcid.org/0000-0002-5835-6945

Dorothee Volkert http://orcid.org/0000-0002-1003-6395

Eva Kiesswetter http://orcid.org/0000-0003-1721-215X

\section{REFERENCES}

1 WHO. Obesity and overweight. Available: https://www.who.int/ news-room/fact-sheets/detail/obesity-and-overweight [Accessed 08 Aug 2020].

2 GBD 2015 Obesity Collaborators, Afshin A, Forouzanfar MH, et al Health effects of overweight and obesity in 195 countries over 25 years. N Engl J Med 2017;377:13-27.

3 He W, Goodkind D, Kowal PUS. P95/16-1, an aging world: 2015, US. Washington, DC: Government Publishing Office, 2016.

4 Hales CM, Fryar CD, Carroll MD, et al. Trends in obesity and severe obesity prevalence in US youth and adults by sex and age, 20072008 to 2015-2016. JAMA 2018;319:1723-5.

5 Batsis JA, Mackenzie TA, Bartels SJ, et al. Diagnostic accuracy of body mass index to identify obesity in older adults: NHANES 1999-2004. Int J Obes 2016;40:761-7.

6 Germain CM, Vasquez E, Batsis JA. Physical activity, central adiposity, and functional limitations in community-dwelling older adults. J Geriatr Phys Ther 2016;39:71-6.

7 Kemmler W, Teschler M, Weißenfels A, et al. Prevalence of sarcopenia and sarcopenic obesity in older German men using recognized definitions: high accordance but low overlap! Osteoporos Int 2017;28:1881-91.

8 Kemmler W, von Stengel S, Engelke K, et al. Prevalence of sarcopenic obesity in Germany using established definitions: baseline data of the formosa study. Osteoporos Int 2016;27:275-81.

9 Batsis JA, Barre LK, Mackenzie TA, et al. Variation in the prevalence of sarcopenia and sarcopenic obesity in older adults associated with different research definitions: dual-energy X-ray absorptiometry data from the National health and nutrition examination survey 1999-2004. J Am Geriatr Soc 2013;61:974-80. 
10 Atkins JL, Whincup PH, Morris RW, et al. Sarcopenic obesity and risk of cardiovascular disease and mortality: a population-based cohort study of older men. J Am Geriatr Soc 2014;62:253-60.

11 The Global BMI Mortality Collaboration. Body-Mass index and all-cause mortality: individual-participant-data meta-analysis of 239 prospective studies in four continents. Lancet 2016;388:776-86.

12 Laxy M, Teuner C, Holle R, et al. The association between BM and health-related quality of life in the US population: sex, age and ethnicity matters. Int J Obes 2018;42:318-26.

13 Cho Y, Shin S-Y, Shin M-J. Sarcopenic obesity is associated with lower indicators of psychological health and quality of life in Koreans. Nutr Res 2015;35:384-92.

14 Greenberg JA. The obesity paradox in the US population. Am J Clin Nutr 2013;97:1195-200.

15 Romero-Corral A, Montori VM, Somers VK, et al. Association of bodyweight with total mortality and with cardiovascular events in coronary artery disease: a systematic review of cohort studies. Lancet 2006;368:666-78.

16 Whelton SP, McAuley PA, Dardari Z, et al. Association of BMI, fitness, and mortality in patients with diabetes: evaluating the obesity paradox in the Henry Ford exercise testing project (fit project) cohort. Diabetes Care 2020;43:677-82.

17 Badrick E, Sperrin M, Buchan IE, et al. Obesity paradox and mortality in adults with and without incident type 2 diabetes: a matched population-level cohort study. BMJ Open Diab Res Care 2017;5:e000369.

18 Banack HR, Kaufman JS. The "Obesity Paradox" Explained. Epidemiology 2013;24:461-2.

19 Preston SH, Stokes A. Obesity paradox: conditioning on disease enhances biases in estimating the mortality risks of obesity. Epidemiology 2014;25:454-61.

20 Villareal DT, Apovian CM, Kushner RF, et al. Obesity in older adults: technical review and position statement of the American Society for nutrition and NAASO, the obesity Society. Obes Res 2005;13:1849-63.

21 Silverwood V, Blagojevic-Bucknall M, Jinks C, et al. Current evidence on risk factors for knee osteoarthritis in older adults: a systematic review and meta-analysis. Osteoarthritis Cartilage 2015;23:507-15

22 Cross M, Smith E, Hoy D, et al. The global burden of hip and knee osteoarthritis: estimates from the global burden of disease 2010 study. Ann Rheum Dis 2014;73:1323-30.

23 Schaap LA, Koster A, Visser M. Adiposity, muscle mass, and muscle strength in relation to functional decline in older persons. Epidemiol Rev 2013;35:51-65.

24 Guralnik JM, Ferrucci L, Pieper CF, et al. Lower extremity function and subsequent disability: consistency across studies, predictive models, and value of gait speed alone compared with the short physical performance battery. J Gerontol A Biol Sci Med Sci 2000;55:M221-31.

25 Guralnik JM, Simonsick EM, Ferrucci L, et al. A short physical performance battery assessing lower extremity function: association with self-reported disability and prediction of mortality and nursing home admission. J Gerontol 1994;49:M85-94.

26 Baumgartner RN, Wayne SJ, Waters DL, et al. Sarcopenic obesity predicts instrumental activities of daily living disability in the elderly. Obes Res 2004;12:1995-2004.

27 Rolland Y, Lauwers-Cances V, Cristini C, et al. Difficulties with physical function associated with obesity, sarcopenia, and sarcopenic-obesity in community-dwelling elderly women: the EPIDOS (EPIDemiologie de l'OSteoporose) Study. Am J Clin Nutr 2009;89:1895-900.

28 Himes CL, Reynolds SL. Effect of obesity on falls, injury, and disability. J Am Geriatr Soc 2012;60:124-9.

29 Scott D, Chandrasekara SD, Laslett LL, et al. Associations of sarcopenic obesity and Dynapenic obesity with bone mineral density and incident fractures over $5-10$ years in communitydwelling older adults. Calcif Tissue Int 2016;99:30-42.

30 Follis S, Cook A, Bea JW, et al. Association between sarcopenic obesity and falls in a multiethnic cohort of postmenopausal women. J Am Geriatr Soc 2018;66:2314-20.

31 Scott D, Seibel M, Cumming R, et al. Sarcopenic obesity and its temporal associations with changes in bone mineral density, incident falls, and fractures in older men: the Concord health and ageing in men project. J Bone Miner Res 2017;32:575-83.

32 Zizza CA, Herring A, Stevens J, et al. Obesity affects nursingcare facility admission among whites but not blacks. Obes Res 2002;10:816-23.

33 Alley DE, Ferrucci L, Barbagallo $M$, et al. A research agenda: the changing relationship between body weight and health in aging. $J$ Gerontol A Biol Sci Med Sci 2008;63:1257-9.
34 Fried LP, Tangen CM, Walston J, et al. Frailty in older adults: evidence for a phenotype. J Gerontol A Biol Sci Med Sci 2001;56:M146-57.

35 Rockwood K, Stadnyk K, MacKnight C, et al. A brief clinical instrument to classify frailty in elderly people. Lancet 1999;353:205-6.

36 Kent S, Fusco F, Gray A, et al. Body mass index and healthcare costs: a systematic literature review of individual participant data studies. Obes Rev 2017;18:869-79.

37 Calculating the costs of the consequences of obesity, 2017. Available: https://www.worldobesity.org/resources/resource-library/ calculating-the-costs-of-the-consequences-of-obesity [Accessed 08 Aug 2020].

38 Alfadda A, Al-Dhwayan M, Alharbi A, et al. The Saudi clinical practice guideline for the management of overweight and obesity in adults. Saudi Med J 2016;37:1151-62.

39 Hauner H, Moss A, Berg A, et al. Interdisziplinäre Leitlinie der Qualität S3 zur „Prävention und Therapie der Adipositas”. Adipositas - Ursachen, Folgeerkrankungen, Therapie 2014:08:179-221.

40 Kushner RF, Ryan DH. Assessment and lifestyle management of patients with obesity: clinical recommendations from systematic reviews. JAMA 2014;312:943-52.

41 Villareal DT, Chode S, Parimi N, et al. Weight loss, exercise, or both and physical function in obese older adults. N Engl J Med 2011;364:1218-29.

42 Porter Starr KN, Pieper CF, Orenduff MC, et al. Improved function with enhanced protein intake per meal: a pilot study of weight reduction in frail, obese older adults. J Gerontol A Biol Sci Med Sci 2016;71:1369-75.

43 Villareal DT, Aguirre L, Gurney AB, et al. Aerobic or resistance exercise, or both, in dieting obese older adults. $N$ Engl J Med 2017;376:1943-55.

44 Miller GD, Nicklas BJ, Davis C, et al. Intensive weight loss program improves physical function in older obese adults with knee osteoarthritis. Obesity 2006;14:1219-30.

45 Cetin DC, Nasr G. Obesity in the elderly: more complicated than you think. Cleve Clin J Med 2014;81:51-61.

46 Weinheimer EM, Sands LP, Campbell WW. A systematic review of the separate and combined effects of energy restriction and exercise on fat-free mass in middle-aged and older adults: implications for sarcopenic obesity. Nutr Rev 2010;68:375-88.

47 Soltani S, Hunter GR, Kazemi A, et al. The effects of weight loss approaches on bone mineral density in adults: a systematic review and meta-analysis of randomized controlled trials. Osteoporos Int 2016;27:2655-71.

48 Mathus-Vliegen EMH, Obesity Management Task Force of the European Association for the Study of Obesity. Prevalence, pathophysiology, health consequences and treatment options of obesity in the elderly: a guideline. Obes Facts 2012;5:460-83.

49 Volkert D, Beck AM, Cederholm T, et al. ESPEN guideline on clinical nutrition and hydration in geriatrics. Clin Nutr 2019;38:10-47.

50 Brawley LR, Rejeski WJ, King AC. Promoting physical activity for older adults: the challenges for changing behavior. Am J Prev Med 2003;25:172-83.

51 American College of Cardiology/American Heart Association Task Force on Practice Guidelines, Obesity Expert Panel, 2013. Expert panel report: guidelines (2013) for the management of overweight and obesity in adults. Obesity 2014;22 Suppl 2:S41-410.

52 Jensen MD, Ryan DH, Apovian CM, et al. 2013 AHA/ACC/TOS guideline for the management of overweight and obesity in adults: a report of the American College of Cardiology/American heart association Task force on practice guidelines and the obesity Society. Circulation 2014;129:S102-38.

53 Bales CW, Buhr G. Is obesity bad for older persons? A systematic review of the pros and cons of weight reduction in later life. J Am Med Dir Assoc 2008;9:302-12.

54 Batsis JA, Gill LE, Masutani RK, et al. Weight loss interventions in older adults with obesity: a systematic review of randomized controlled trials since 2005. J Am Geriatr Soc 2017;65:257-68.

55 Bouaziz W, Schmitt E, Kaltenbach G, et al. Health benefits of endurance training alone or combined with diet for obese patients over 60: a review. Int J Clin Pract 2015;69:1032-49.

56 Felix HC, West DS. Effectiveness of weight loss interventions for obese older adults. Am J Health Promot 2013:27:191-9.

57 Haywood C, Sumithran P. Treatment of obesity in older persons-A systematic review. Obes Rev 2019;20:588-98.

58 McTigue KM, Hess R, Ziouras J. Obesity in older adults: a systematic review of the evidence for diagnosis and treatment. Obesity 2006;14:1485-97. 
59 Poggiogalle E, Migliaccio S, Lenzi A, et al. Treatment of body composition changes in obese and overweight older adults: insight into the phenotype of sarcopenic obesity. Endocrine 2014;47:699-716.

60 Porter Starr KN, McDonald SR, Bales CW. Obesity and physical frailty in older adults: a scoping review of lifestyle intervention trials. J Am Med Dir Assoc 2014;15:240-50.

61 Rejeski WJ, Marsh AP, Chmelo E, et al. Obesity, intentional weight loss and physical disability in older adults. Obes Rev 2010;11:671-85.

62 Waters DL, Ward AL, Villareal DT. Weight loss in obese adults 65years and older: a review of the controversy. Exp Gerontol 2013;48:1054-61.

63 Witham MD, Avenell A. Interventions to achieve long-term weight loss in obese older people: a systematic review and meta-analysis. Age Ageing 2010;39:176-84.

64 Greaves C, Poltawski L, Garside R, et al. Understanding the challenge of weight loss maintenance: a systematic review and synthesis of qualitative research on weight loss maintenance. Health Psychol Rev 2017;11:145-63.

65 Brassey J, Spencer EA, Heneghan C, Catalogue of Bias Collaboration. Langauge bias. In: Catalogue of bias, 2017. https:// catalogofbias.org/biases/language-bias/

66 Puljak L. If there is only one author or only one database was searched, a study should not be called a systematic review. J Clin Epidemiol 2017;91:4-5.

67 Ard JD, Gower B, Hunter G, et al. Effects of calorie restriction in obese older adults: the crossroads randomized controlled trial. $J$ Gerontol A Biol Sci Med Sci 2017;73:73-80.

68 Beavers KM, Nesbit BA, Kiel JR, et al. Effect of an EnergyRestricted, nutritionally complete, higher protein meal plan on body composition and mobility in older adults with obesity: a randomized controlled trial. J Gerontol A Biol Sci Med Sci 2019;74:929-35.

69 Cumpston M, Li T, Page MJ, et al. Updated guidance for trusted systematic reviews: a new edition of the Cochrane Handbook for systematic reviews of interventions. Cochrane Database Syst Rev 2019;10:ED000142.

70 Higgins JPT TJ, Chandler J, Cumpston M, et al, eds. Cochrane Handbook for Systematic Reviews of Interventions. 2nd ed. Chichester (UK):: John Wiley \& Sons, 2019.

71 Jackson SE, Holter L, Beeken RJ. 'Just because I'm old it doesn't mean I have to be fat': a qualitative study exploring older adults views and experiences of weight management. BMJ Open 2019;9:e025680-e80.

72 Moher D, Shamseer L, Clarke M, et al. Preferred reporting items for systematic review and meta-analysis protocols (PRISMA-P) 2015 statement. Syst Rev 2015;4:1.

73 Chaimani A, Caldwell DM, Li T, et al. Additional considerations are required when preparing a protocol for a systematic review with multiple interventions. J Clin Epidemiol 2017;83:65-74.

74 Shenkin SD, Harrison JK, Wilkinson T, et al. Systematic reviews: guidance relevant for studies of older people. Age Ageing 2017:46:722-8.

75 Torbahn G, Schoene D, Schwingshackl L, et al. EffectiveSLOPE effects of lifestyle interventions in obese older people - a systematic review and network meta-analysis. prospero 2019 CRD42019147286, 2019. Available: https://www.crd.york.ac.uk/prospero/display_record. php?ID=CRD42019147286 [Accessed 08 Aug 2020].

76 NHS England. Improving care for older people. Available: https:// www.england.nhs.uk/ourwork/clinical-policy/older-people/ improving-care-for-older-people/ [Accessed 08 Aug 2020].

77 World Health Organisation. Physical status: the use and interpreation of anthropometry. technical report series 854. Geneva: WHO, 1995.

78 Han TS, van Leer EM, Seidell JC, et al. Waist circumference action levels in the identification of cardiovascular risk factors: prevalence study in a random sample. BMJ 1995;311:1401-5.

79 Batsis JA, Zagaria AB. Addressing obesity in aging patients. Med Clin North Am 2018;102:65-85.

80 Valiyeva E, Russell LB, Miller JE, et al. Lifestyle-related risk factors and risk of future nursing home admission. Arch Intern Med 2006;166:985-90.

81 Astbury NM, Piernas C, Hartmann-Boyce J, et al. A systematic review and meta-analysis of the effectiveness of meal replacements for weight loss. Obes Rev 2019;20:569-87.

82 American College of Sports Medicine, Chodzko-Zajko WJ, Proctor DN, et al. American College of sports medicine position stand. exercise and physical activity for older adults. Med Sci Sports Exerc 2009;41:1510-30.
83 National Health and Medical Research Council. Clinical practice guidelines for the management of overweight and obesity in adults, adolescents and children in Australia. Melbourne: National Health and Medical Research Council, 2013. https://www.nhmrc.gov.au/ about-us/publications/clinical-practice-guidelines-managementoverweight-and-obesity\#block-views-block-file-attachmentscontent-block-1

84 Wong SKW, Smith HE, Chua JJS, et al. Effectiveness of selfmanagement interventions in young adults with type 1 and 2 diabetes: a systematic review and meta-analysis. Diabet Med 2020;37:229-41.

85 Stutzer A, Meier AN. Limited self-control, obesity, and the loss of Happiness. Health Econ 2016;25:1409-24.

86 Teixeira PJ, Carraça EV, Marques MM, et al. Successful behavior change in obesity interventions in adults: a systematic review of self-regulation mediators. BMC Med 2015;13:84.

87 Reuben DB, Siu AL. An objective measure of physical function of elderly outpatients. The physical performance test. J Am Geriatr Soc 1990;38:1105-12.

88 Ware JE, Sherbourne CD. The mos 36-item short-form health survey (SF-36). I. conceptual framework and item selection. Med Care 1992;30:473-83.

89 EuroQol Group. EuroQol - a new facility for the measurement of health-related quality of life. Health Policy 1990;16:199-208.

90 Mead E, Brown T, Rees K, et al. Diet, physical activity and behavioural interventions for the treatment of overweight or obese children from the age of 6 to 11 years. Cochrane Database Syst Rev 2017;6:CD012651.

91 Recker RR, Davies KM, Dowd RM, et al. The effect of low-dose continuous estrogen and progesterone therapy with calcium and vitamin D on bone in elderly women. A randomized, controlled trial. Ann Intern Med 1999;130:897-904.

92 Sterne JAC, Savović J, Page MJ, et al. Rob 2: a revised tool for assessing risk of bias in randomised trials. BMJ 2019;366:14898-198.

93 Reid IR, Ames RW, Evans MC, et al. Long-Term effects of calcium supplementation on bone loss and fractures in postmenopausal women: a randomized controlled trial. Am J Med 1995;98:331-5.

94 Brignardello-Petersen R, Bonner A, Alexander PE, et al. Advances in the GRADE approach to rate the certainty in estimates from a network meta-analysis. J Clin Epidemiol 2018;93:36-44.

95 Puhan MA, Schünemann HJ, Murad MH, et al. A grade Working group approach for rating the quality of treatment effect estimates from network meta-analysis. BMJ 2014;349:g5630.

96 Yepes-Nuñez JJ, Li S-A, Guyatt G, et al. Development of the summary of findings table for network meta-analysis. J Clin Epidemiol 2019;115:1-13.

97 Deng HW, Stegman MR, Davies KM, et al. Genetic determination of variation and covariation of peak bone mass at the hip and spine. $J$ Clin Densitom 1999;2:251-63.

98 Anzures-Cabrera J, Sarpatwari A, Higgins JPT. Expressing findings from meta-analyses of continuous outcomes in terms of risks. Stat Med 2011;30:2967-85

99 Deeks JJ, Higgins JPT, Altman DG. Chapter 10: Analysing data and undertaking meta-analyses. In: Higgins JPT, Thomas J, Chandler J, et al, eds. Cochrane Handbook for systematic reviews of interventions version 6.0 (updated July 2019). Cochrane, 2019. www.training.cochrane.org/handbook

100 Rücker G. Network meta-analysis, electrical networks and graph theory. Res Synth Methods 2012;3:312-24.

101 Stern Y, Tang MX, Albert MS, et al. Predicting time to nursing home care and death in individuals with Alzheimer disease. JAMA 1997;277:806-12.

102 Salanti G. Indirect and mixed-treatment comparison, network, or multiple-treatments meta-analysis: many names, many benefits, many concerns for the next generation evidence synthesis tool. Res Synth Methods 2012;3:80-97.

103 Rücker G, Schwarzer G. Automated drawing of network plots in network meta-analysis. Res Synth Methods 2016;7:94-107.

104 Krahn U, Binder H, König J. A graphical tool for locating inconsistency in network meta-analyses. BMC Med Res Methodol 2013;13:35.

105 Pillay J, Armstrong MJ, Butalia S, et al. Behavioral programs for type 2 diabetes mellitus: a systematic review and network metaanalysis. Ann Intern Med 2015;163:848-60.

106 Rücker G, Petropoulou M, Schwarzer G. Network meta-analysis of multicomponent interventions. Biom J 2020;62:808-21.

107 Rücker G, Schwarzer G. Ranking treatments in frequentist network meta-analysis works without resampling methods. BMC Med Res Methodol 2015;15:58. 